\title{
Pregnancy Outcome in Women with Polycystic Ovary Syndrome
}

\author{
Amandeep Mann $^{1} \cdot$ Haritha Sagili $^{2}$ - Murali Subbaiah ${ }^{1}$ \\ Received: 31 March 2020 / Accepted: 8 July 2020 / Published online: 17 July 2020 \\ (c) Federation of Obstetric \& Gynecological Societies of India 2020
}

\begin{abstract}
Background/Purpose Pregnant women with polycystic ovary syndrome seem to be prone for adverse maternal and perinatal outcomes, but there is no conclusive evidence. Indian data evaluating the pregnancy outcome in women with polycystic ovary syndrome are sparse. This study was proposed to evaluate the pregnancy outcome in women with polycystic ovary syndrome. Methods This descriptive study on 135 pregnant women with polycystic ovary syndrome was carried out in a tertiary care hospital in South India from January 2016 to October 2017. Data regarding present pregnancy, polycystic ovary syndrome and maternal/ perinatal outcomes were analysed using SPSS version 20. Categorical variables studied were parity, various maternal and perinatal outcomes.

Results The mean age was 26.8 years, $77 \%$ had high BMI, and $88 \%$ had history of primary infertility. The mean age was 26.8 years, $77 \%$ had high BMI, and $88 \%$ had history of primary infertility. The proportion of hypertensive disorders of pregnancy was (17.8\%), PROM (18.5\%), low APGAR score at 5 min (13\%), gestational diabetes (13\%), miscarriage (2.2\%), preterm delivery $(10.4 \%)$, caesarean delivery (30.4\%), low birth weight babies (2\%), macrosomia (0.7\%), PPROM (8\%), perinatal mortality $(2 \%)$ and NICU admission (20\%).

Conclusion The proportion of hypertensive disorders of pregnancy, PROM, low birth weight babies, low APGAR score at 5 min was found to be higher, but the proportion of GDM, miscarriage, preterm delivery, meconium stained liquor, caesarean delivery, small for gestational age/IUGR, macrosomia, PPROM, perinatal mortality, NICU admission and congenital anomalies was found to be either similar or lower in pregnant women with PCOS in our study to those described in the general pregnant population.
\end{abstract}

Keywords PCOS $\cdot$ Pregnancy outcome $\cdot$ Maternal outcome $\cdot$ Perinatal outcome

\section{Introduction}

Polycystic ovary syndrome (PCOS) is the most common endocrine disorder in women of reproductive age. It is estimated that 6 to $15 \%$ of women suffer from this disease [1],

Dr. Amandeep Mann, MS in Department of Obstetrics and Gynaecology, JIPMER, Puducherry, India. Dr. Haritha Sagili, MD, MRCOG, MFSRH, Additional Professor in Department of Obstetrics and Gynaecology, JIPMER, Puducherry, India. Dr. Murali Subbaiah, MS, Associate Professor in Department of Obstetrics and Gynaecology, JIPMER, Puducherry, India.

Haritha Sagili

harithasagili@gmail.com

1 Department of Obstetrics and Gynaecology, JIPMER, Puducherry 605006, India

2 Department of Obstetrics and Gynaecology, JIPMER, Puducherry 605005, India diagnosed by any two of the following three criteria: menstrual irregularity with oligo-anovulation, hyperandrogenism and polycystic ovarian features on ultrasound [2]. Pregnant women with PCOS seem to be prone for adverse maternal and perinatal outcomes [1, 3-13]. Adverse maternal outcomes include high chance of miscarriage, increased risk of gestational diabetes mellitus, high risk of development of hypertensive disorders of pregnancy and appear to be more prone for preterm delivery and operative delivery.

Perinatal risks described in the literature are small for gestational age babies, macrosomia, meconium aspiration syndrome, low APGAR score at $5 \mathrm{~min}$, NICU admission and perinatal mortality. One study has shown that the PCOS pregnant women have a high chance of developing congenital anomalies [4] although this has not been shown in other studies. But some studies have also shown either no difference or lower incidence of specific outcomes when compared to women without PCOS [3-13]. Thus, there is no 
conclusive evidence regarding the outcome of these pregnancies. Indian data evaluating the pregnancy outcome in women with polycystic ovary syndrome are sparse $[11,13]$ and are of small sample size. Thus, this study was proposed to evaluate the pregnancy outcomes in women with PCOS.

\section{Materials and Methods}

This descriptive study was carried out in the Department of Obstetrics and Gynaecology, JIPMER, Puducherry, from January 2016 to October 2017. The study protocol was approved by Postgraduate Research Monitoring Committee [No: PGMRC/OG/1/2015] and Ethics Committee of JIPMER [IEC/SC/2015/23/845]. Women diagnosed with PCOS according to Rotterdam's criteria who were willing to participate in our study were recruited from obstetrics OPD and antenatal ward of JIPMER. After obtaining an informed consent from them, a detailed interview schedule containing socio- demographic details, menstrual/marital/ obstetric/past/personal/ family history was taken. A detailed history regarding PCOS, time of diagnosis of polycystic ovary syndrome, criteria considered such as oligomenorrhea, ultrasound findings and testosterone levels in women with PCOS was noted. History of infertility and treatment taken for PCOS was recorded. Physical examination including height, weight, BMI, blood pressure, acanthosis nigricans and hirsutism was noted. The pregnancy was followed up monthly up to delivery. Maternal complications, mode of delivery and perinatal outcome were recorded as mentioned below.

Data were entered in MS Excel and analysed using SPSS version 20. Continuous variables included age, weight, gestational age and were expressed as mean $\pm \mathrm{SD}$ as appropriate. Categorical variables studied were parity, various maternal and perinatal outcomes, and data were expressed as proportions.

\section{Results}

Total number of pregnant women with PCOS who delivered in JIPMER and participated in our study were 135 . The mean age of women in our study was 26.83 years. The percentage of elderly pregnant women in our study was 3.7\%, and there were no teenage pregnancies. Majority (52\%) had finished high school, 29\% had completed higher secondary, and $19 \%$ were graduates. Out of total 135 participants, majority (67\%) belonged to low class, $28 \%$ were in middle class, and $5 \%$ belonged to high class according to modified Prasad's classification. Mean height, weight and BMI were $155 \mathrm{~cm}, 66.69 \mathrm{~kg}$ and $27.15 \mathrm{~kg} / \mathrm{m}^{2}$, respectively. Sixty-three percent $(63 \%)$ of the study population were over-weight in our study, and $15 \%$ were obese. The proportion of Acanthosis nigricans was found to be $5.2 \%$ among study population.

Fifty-three percent (53\%) of women presented with history of infrequent menstrual cycles, and $42 \%$ had polycystic ovaries on ultrasound findings. Eleven percent (11\%) had features of clinical and/or biochemical signs of hyperandrogenism according to Rotterdam's criteria for the diagnosis of PCOS. Almost $94 \%$ of the women had undergone treatment for PCOS prior to conception. Majority 57 (42\%) of women had conceived on metformin, and $9(7 \%)$ women had undergone ovarian drilling.

Majority $(88 \%)$ of the women had primary infertility, and $12 \%$ had secondary infertility and were primigravida. Almost $62 \%$ of women conceived spontaneously, while $38 \%$ women conceived after treatment either with ovulation inducing drugs or intrauterine insemination/in vitro fertilization. Nine percent ( $9 \%$ ) was twins, $0.7 \%$ triplets, and $90.3 \%$ were singleton pregnancies in our study.

Maternal outcome of these pregnancies is summarized in Table 1. Fourteen percent (14\%) of women had gestational hypertension, and $4 \%$ had pre-eclampsia. Thirteen percent

Table 1 Maternal outcome in study population

\begin{tabular}{|c|c|c|}
\hline Characteristic & Frequency $(n)$ & Percentage $(\%)$ \\
\hline \multicolumn{3}{|l|}{ Maternal outcomes } \\
\hline \multicolumn{3}{|l|}{ Medical } \\
\hline \multicolumn{3}{|l|}{ Hypertension } \\
\hline Gestational hypertension & 19 & 14.1 \\
\hline Pre-eclampsia & 5 & 3.7 \\
\hline \multicolumn{3}{|l|}{ Diabetes } \\
\hline GDMA1 & 8 & 5.9 \\
\hline GDMA2 & 10 & 7.4 \\
\hline \multicolumn{3}{|l|}{ Obstetric } \\
\hline Abortion & 3 & 2.2 \\
\hline IUGR/ SGA & 3 & 2.2 \\
\hline \multicolumn{3}{|l|}{ Rupture of membranes } \\
\hline PPROM & 11 & 8.1 \\
\hline PROM & 25 & 18.5 \\
\hline Preterm labour & 14 & 10.4 \\
\hline \multicolumn{3}{|l|}{ Delivery } \\
\hline \multicolumn{3}{|l|}{ Period of gestation } \\
\hline Preterm & 14 & 10.4 \\
\hline Term & 119 & 88.1 \\
\hline Post-term & 2 & 1.5 \\
\hline \multicolumn{3}{|l|}{ Nature of labour } \\
\hline Induced & 68 & 50.5 \\
\hline Spontaneous & 58 & 6.6 \\
\hline \multicolumn{3}{|l|}{ Mode of delivery } \\
\hline SVD & 80 & 59.2 \\
\hline Instrumental delivery & 14 & 10.4 \\
\hline Caesarean section & 41 & 30.4 \\
\hline
\end{tabular}


(13\%) of women were diagnosed to have gestational diabetes mellitus out of which $6 \%$ were on diabetic diet and $7 \%$ needed metformin/insulin. Two women had spontaneous miscarriages, one in the second trimester and the other in the first trimester. One patient had an induced abortion in second trimester at 19 weeks due to cystic hygroma. Almost $27 \%$ of the participants presented with rupture of membranes, out of which $19 \%$ had rupture of membrane at term gestation and $8 \%$ had preterm rupture of membranes. Approximately $10 \%$ of women had preterm labour. $2.2 \%$ of the women who delivered at term had small for gestational age babies.

Ten percent $(10 \%)$ of women were preterm at the time of delivery, and $2 \%$ were post-term. Half the women $(50 \%)$ underwent induction of labour; $43 \%$ went into spontaneous labour; and 7\% had elective LSCS. Majority of the women $(59 \%)$ were delivered by spontaneous vaginal delivery. Ten percent (10)\% of women were delivered by instrumental delivery and $30 \%$ by caesarean section.

Perinatal outcome of pregnant women with PCOS is shown in Table 2. Majority of the babies (97\%) were born healthy and alive. Two percent $(2 \%)$ were still born, out of which one mother had gestational diabetes with hypertension. One baby died on postnatal day 5 due to respiratory distress with low birth weight. Majority of the babies born had weight in the range of $\leq 1,000 \mathrm{~g}(3 \%), 2,500-2,999 \mathrm{~g}$ (31\%), 26\% babies had weight in the range of 3,000-3,499, and $7.4 \%$ were $\geq 3,500 \mathrm{~g}$. Majority of the babies (86\%) were

Table 2 Perinatal outcome

\begin{tabular}{|c|c|c|}
\hline \multicolumn{3}{|l|}{ Status of newborn } \\
\hline Alive & 146 & 97.8 \\
\hline Still birth & 2 & 1.5 \\
\hline Neonatal death & 1 & 0.7 \\
\hline \multicolumn{3}{|c|}{ Birth weight of newborn (grams) } \\
\hline$<1000$ & 4 & 2.7 \\
\hline $1001-1499$ & 9 & 6.0 \\
\hline $1500-1999$ & 14 & 9.4 \\
\hline $2000-2499$ & 27 & 18.1 \\
\hline $2500-2999$ & 46 & 30.9 \\
\hline $3000-3499$ & 38 & 25.5 \\
\hline $3500-3999$ & 10 & 6.7 \\
\hline$>4000$ & 1 & 0.7 \\
\hline Characteristic & Frequency $(n)$ & Percentage $(\%)$ \\
\hline \multicolumn{3}{|l|}{ APGAR score } \\
\hline $0 / 0$ & 2 & 1.3 \\
\hline$<8 / 9$ & 19 & 12.8 \\
\hline$>$ or $=8 / 9$ & 128 & 85.9 \\
\hline Meconium stained liquor & 11 & 7.4 \\
\hline NICU admission & 29 & 19.5 \\
\hline Congenital anomaly & 2 & 1.3 \\
\hline
\end{tabular}

born with APGAR value $\geq 8 / 9$. Thirteen percent (13\%) had low APGAR score due to respiratory distress. Meconium stained liquor was observed in $7.4 \%$ of our study population, and there were no cases of meconium aspiration in our study. Almost $20 \%$ of the babies were admitted to NICU either due to low birth weight or respiratory distress. Out of NICU admission, 15 (10.06\%) babies required bag and mask ventilation and 3 babies were intubated. Out of 149 foetuses delivered, 2 (1.3\%) were diagnosed to have anomalies. One foetus had cystic hygroma, and the pregnancy was terminated at 19 weeks. The other baby, second of twin pregnancy was delivered at 37 weeks of gestation by spontaneous vaginal delivery, diagnosed to have hypoplasia of lungs postnatally and was on ventilatory support. The baby was taken home against medical advice.

\section{Discussion}

There are several studies which have evaluated pregnancy outcomes in women with PCOS, but the findings are inconclusive. The mean age of women in our study was 26.8 years with age distribution ranging from 21 to 44 years. The elderly pregnant women were $3.7 \%$, and there were no teenage pregnancies which is comparable to other studies [4, 5, 8, 9, 11, 12]. Majority of the pregnant women with PCOS in our study (52\%) had finished high school, 29\% had completed higher secondary, and 19\% were graduates which is similar to another study [9].

The mean BMI among women pregnant with PCOS in our study group was $27.15 \mathrm{~kg} / \mathrm{m}^{2}$. A higher mean BMI of $30.8 \mathrm{~kg} / \mathrm{m}^{2}$ was seen in overweight women with PCOS when compared to normal weight women in another retrospective cohort study [5]. Sixty-three percent of the study population were over-weight in our study, and $15 \%$ were obese which is lower when compared to another population-based study which had $61 \%$ prevalence of obesity [9]. A small retrospective Indian study in 110 pregnant women with PCOS showed a lower prevalence of overweight women $(58 \%)$ in their results [12].

The proportion of Acanthosis nigricans in women with PCOS in our study was $5.2 \%$, and $80.5 \%$ women were diagnosed to have hypothyroidism. There is no mention of Acanthosis in other studies. Primigravida in our study were $88.1 \%$, which is much higher when compared to other studies, which had a prevalence ranging from 47 to $81 \%$ $[5,9,11]$. Almost $62 \%$ of women conceived spontaneously, while $38 \%$ women conceived after treatment either with ovulation inducing drugs or assisted reproductive technology in our study. This is in contrast to other studies which had a lower rate of spontaneous conception of $29 \%$ [11] and a higher pregnancy rate of $71.4 \%$ with use of OI and ART [13]. In another Australian study in 2,566 PCOS 
women the proportion of women who had conceived after in vitro fertilization was $8 \%$ similar to our study [4].

In pregnant women with PCOS the proportion of multifoetal pregnancy was $10 \%$ in our study which is similar to a study done in Finland on 99 PCOS women [14]. But it is much higher when compared to an Australian study (3.3\%) [4]. The proportion of hypertension and preeclampsia in our study of pregnant women with PCOS was found to be $14.1 \%$ and $3.7 \%$, respectively, similar to other studies [3, $11,13]$. However, a lower incidence of hypertension $2.4 \%$ to $11 \%[3,5,12]$ and higher percentage of preeclampsia of $8-12 \%$ has also been demonstrated [4, 5]. A 2-fourfold increase in hypertension/preeclampsia has been described in five metaanalyses on pregnancy outcome in women with PCOS [6-10].

Diagnosis of GDM during pregnancy in women with PCOS in our study was $13 \%$ which is similar to another Indian case-control study on 56 PCOS women [11]. But a lower incidence of GDM ranging from $7.2 \%$ to $8 \%$ has been found in other studies $[4,5,13]$. Two other studies in contrast showed a higher incidence of GDM of $22 \%$ in PCOS pregnant women [3, 12]. A two-threefold increased risk of GDM in pregnant women with PCOS is described in metaanalyses so far [6-10]. The percentage of miscarriage in the present pregnancy in women with PCOS in our study was $2.2 \%$, which is less as compared with previous studies which found an incidence ranging from $8.1 \%$ to as high as $20 \%[4,12,13]$.

The proportion of SGA/IUGR babies in women with PCOS in our study was $2 \%$ similar to another Indian retrospective study [12]. This is lesser when compared to other studies which found a higher incidence of SGA/IUGR babies ranging from $8 \%$ to $13 \%[3,4]$. No difference in risk of SGA/IUGR has been demonstrated in some studies [6,9]. A 1.5-twofold increased risk has been found in some studies when compared to normal pregnant women $[8,10,15]$. The women who had rupture of membranes at preterm or term gestation in our study was $8 \%$ and $18.5 \%$, respectively. The incidence of rupture of membranes at term was much higher compared to another Indian retrospective study on 110 PCOS women of $8 \%$ [12], whereas the incidence of preterm rupture of membranes was similar to a metaanalysis [8].

The incidence of preterm delivery was $10.4 \%$ in our study, which is similar to one Indian prospective study on 56 PCOS women [13]. This is much lesser when compared to other studies which have found a higher incidence ranging from 13.9 to $19.2 \%$ [3-5, 12]. A 1.3-3.9fold increased risk of preterm delivery in pregnant women with PCOS has been described in several metaanalyses [6-10]. The percentage of women who underwent caesarean delivery or assisted vaginal delivery in our study was $30.4 \%$ and $10.4 \%$, respectively. The incidence of caesarean section was lower in our study when compared to other studies which showed a higher prevalence $39-64 \%$ [3-5, 11, 12]. No difference in risk has been demonstrated in one metaanalysis of 27 studies involving 4982 PCOS women [7]. However, a 1.2-1.9fold increased risk has been shown in other metaanalyses $[8,10]$.

The proportion of macrosomia in our study was $0.7 \%$. According to studies done so far a higher incidence of macrosomia in PCOS pregnant women ranging from 6 to $18 \%$ has been described [3-5, 12]. Women with PCOS have $1.2-1.5$ fold higher chance of having macrosomic babies according to metaanalyses [8-10]. Another prospective study done in India on 56 PCOS women found that the women who were on metformin therapy had no macrosomia babies [13]. In our study the incidence of low birth weight babies, very low birth weight babies, extreme low birth weight babies was $27.4 \%, 6 \%$ and $2.7 \%$, whereas in two other studies the incidence of low birth weight babies was lower $(4.9-11.4 \%)[4,5]$.

In our study $13 \%$ of babies born to pregnant women with PCOS had APGAR of $<8 / 9$ at 5 min which is higher when compared to another Australian study which found an incidence of $4.2 \%$ [4]. But a 1.4fold increased risk of having low APGAR was found in a population-based cohort study on 3787 PCOS women [9]. The incidence of meconium stained liquor in our study was $7.4 \%$ which is slightly higher when compared to other studies which found an incidence ranging from 3.2 to $3.6 \%$ [3, 12]. However, according to two metaanalyses, there is a 1.2-2.3fold increased risk of having meconium stained liquor during delivery $[8,9]$.

In our study (97\%) of babies were born healthy and alive. Two percent were still born, which is similar to an Australian study and another retrospective study $(3 \%)[3,4]$. NICU admission of babies born to pregnant women with PCOS in our study was $20 \%$. A lower incidence of $8 \%-14 \%$ has been described in some studies [3, 4], whereas a higher incidence of $25 \%-30 \%$ has been found in other studies [5, 7, 12]. A 2.3fold higher chance of NICU admission has been reported in a metaanalysis of 27 studies on 4982 PCOS women [7]. In our study the incidence of congenital anomalies was $1.3 \%$ which is almost similar to two other Indian studies and a metaanalysis $[8,12,13]$, but a high prevalence of $6 \%$ was found in a study done in Australia which found that offspring born to PCOS mothers may have cardiovascular and urogenital defects [4]. In our study, one foetus had cystic hygroma and the other baby born to twin gestation mother was diagnosed to have hypoplasia of lungs. The incidence of neonatal deaths in our study was $0.7 \%$ which is similar to another study on 1789 women with PCOS [4], but lower when compared to a retrospective matched cohort study [3]. A 1.5-1.8fold increased risk has been described in two other metaanalyses $[8,9]$.

Thus, overall the proportion of adverse maternal and perinatal outcomes appears to be in agreement with some studies but differs to other trials carried out in pregnant women with 
PCOS. This is one of the very few studies from India with a relatively larger sample size to provide information on the pregnancy outcome in women with PCOS. Also our study included pregnancies that were both spontaneously conceived as well as by assisted reproductive technology and included both singleton and multiple pregnancies. The limitations of the study were that since the study population was recruited from a tertiary hospital, the pregnancy outcome may not reflect all pregnant women with PCOS, and there was no comparison with a control pregnant population without PCOS.

The proportion of hypertensive disorders of pregnancy, PROM, low birth weight babies, low APGAR score at 5 min was found to be higher, but the proportion of GDM, miscarriage, preterm delivery, meconium stained liquor, caesarean delivery, small for gestational age/IUGR, macrosomia, PPROM, perinatal mortality, NICU admission and congenital anomalies was found to be either similar or lower in pregnant women with PCOS in our study to those described in the general pregnant population [16].

\section{Conclusions}

The data in our study throw more light on the current thinking of the maternal and perinatal problems faced by PCOS mothers, in which some of our results support and others refute some of the risks in pregnancy in women with PCOS. Further larger cohort studies with longer follow-up are needed to study the strength of association of PCOS with the conflicting adverse maternal and perinatal outcomes of pregnancy.

\section{Compliance with Ethical Standards}

Conflict of interest The authors Amandeep Mann, Haritha Sagili, Murali Subbaiah report no conflict of interest.

Human and Animal Rights All procedures followed were in accordance with the ethical standards of the responsible committee on human experimentation (institutional and national) and with the Helsinki Declaration of 1975, as revised in 2008 .

Informed Consent Informed consent was obtained from all patients for being included in the study.

\section{References}

1. Kamalanathan S, Sahoo JP, Sathyapalan T. Pregnancy in polycystic ovary syndrome. Indian J Endocr Metab. 2013;17:37-433.

2. Rotterdam ESHRE/ASRM-Sponsored PCOS Consensus Workshop. Revised 2003 consensus on diagnostic criteria and longterm health risks related to polycystic ovary syndrome. FertilSteril. 2004;81:19-25.

3. Kollmann M, Klaritsch P, Martins WP, et al. Maternal and neonatal outcomes in pregnant women with PCOS: comparison of different diagnostic definitions. Hum Reprod. 2015;30(10):2396-403.

4. Doherty DA, Newnham JP, Bower C, et al. Implications of polycystic ovary syndrome for pregnancy and for the health of offspring. Obstet Gynecol. 2015;125(6):1397-406.

5. Frene VD, Vansteelandt S, Sjoen GT, et al. A retrospective study of the pregnancy, delivery and neonatal outcome in overweight versus normal weight women with polycystic ovary syndrome. Hum Reprod. 2014;0(0):1-6.

6. Palomba S, Dewilde MA, Falbo A, et al. Pregnancy complications in women with polycystic ovary syndrome. Hum Reprod. 2015;21(5):575-92.

7. Qin JZ, Pang LH, Li MJ, Fan XJ, Huang RD, Chen HY. Obstetric complications in women with polycystic ovary syndrome: a systematic review and meta-analysis. Reprod Biol Endocrinol. 2013;11:56.

8. Yu HF, Chen HS, Rao DP, et al. Association between polycystic ovary syndrome and the risk of pregnancy complications. A prisma-complaint systemic review and metaanalysis. Medicine. 2016;95:51.

9. Roos N, Kieler H, Sahlin L, et al. Risk of adverse pregnancy outcomes in women with polycystic ovary syndrome: population based cohort study. BMJ. 2011;343:d6309.

10. Kjerulff LE, Sanchez-Ramos L, Duffy D. Pregnancy outcomes in women with polycystic ovary syndrome: a metaanalysis. Am J Obstet Gynecol. 2011;204(6):558.e551-556.

11. Amita G, Kapila R, Tania K, et al. Pregnancy outcome in women with the polycystic ovarian syndrome. JK Sci. 2009;11(2):82-4.

12. Puvithra T, Pandiyan R, Pandiyan N. Pregnancy complicationsconsequence of polycystic ovary syndrome or body mass index. Chettinad Health Med J. 2017;6(1):04-8.

13. Nandita PP, Hrishikesh DP. Metformin throughout pregnancy in women with polycystic ovary syndrome: safety and advantages. Int J Infertil Fetal Med. 2011;2(2):61-4.

14. Mikova M, Hiileesma V, Halttunen M, et al. Obstetrics outcome in women with polycystic ovary syndrome. Hum Reprod. 2001;16(2):226-9.

15. Sir-Petermann T, Hitchsfeld C, Maliqueo M, et al. Birth weight in offspring of mothers with polycystic ovarian syndrome. Hum Reprod. 2005;20:2122-6.

16. Cunningham FG, Leveno JK, Bloom SL, et al. Williams obstetrics. 25th ed. New York: McGraw-Hill; 2018.

Publisher's Note Springer Nature remains neutral with regard to jurisdictional claims in published maps and institutional affiliations. 


\section{About the Author}

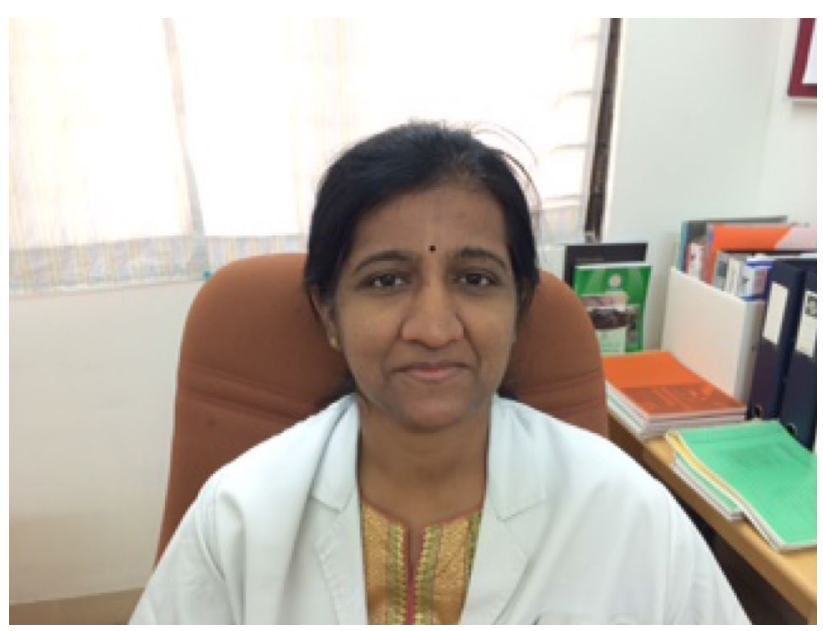

Haritha Sagili completed her postgraduation in Obstetrics and
Gynecology from Jawaharlal Institute of Postgraduate Medical Education and Research, a referral tertiary care and teaching hospital in Puducherry, South India, with an annual delivery rate of 15,000/year. She then pursued her further career in the UK for 4 years where she passed the MRCOG and MFSRH examinations. She joined back her alma mater as an Assistant Professor in 2009 as she is passionate about teaching. She has about 62 publications to her credit and is currently involved in various research projects. 